\title{
sciendo
}

\section{A PILOT STUDY TO EXAMINE THE EFFECTS OF A SCHOOL- BASED INTERVENTION FOR PROMOTING FOOD AND NUTRITION LITERACY (FNLIT) IN ROMANIAN SCHOOL CHILDREN}

\author{
ALINA-IOANA FORRAY ${ }^{1}$, ANCA-GEORGIANA GOLOGAN ${ }^{2}$, MĂDĂLINA COMAN ${ }^{3}$, \\ RĂZVAN-MIRCEA CHERECHEȘ ${ }^{4}$, DUMITRU PETREUS ${ }^{5}$, SVEATOSLAV VIZITIU $^{6}$, \\ CRISTINA-MARIA BORZAN ${ }^{7}$
}

\begin{abstract}
1,7 "Iuliu Hațieganu" University of Medicine and Pharmacy, ${ }^{1,2,3,4}$ "Babeș-Bolyai" University, ${ }^{5,6}$ Wello Association
Keywords: $\quad$ nutrition, Abstract: The prevalence of Non-Communicable Diseases (NCDs) is an important public health education, food and problem in Romania, where, nutrition transition has taken place and has resulted in a more nutrition literacy, school- westernized dietary pattern, especially among children and adolescents. WHO has identified schools based intervention, health as the ideal place to educate children and adolescents efficiently in Food and Nutrition Literacy promotion (FNLIT). The present research consisted of a pilot study to determine the effectiveness of a nutrition education programme implemented among lower secondary school children by investigating whether students tended to score higher in several FNLIT domains at post-test compared to the pre-test average. The implementation of the intervention consisted of five lessons in online or physical classes. Even if the intervention period had a relatively short period of implementation improvements in understanding, interactive food and nutrition literacy, critical food and label literacy were observed. Future research should focus on expanding the intervention in other schools.
\end{abstract}

\section{INTRODUCTION}

The prevalence of Non-Communicable Diseases (NCDs) is an important public health problem in Romania. Prevention of NCDs is an important priority that needs to be addressed by encouraging the population to have control over the determinants of their health. $(1,2)$ Health literacy (HL) is a predictor that plays an important role in the prevention of NCDs. Lower levels of HL were identified as an important contributor to the burden of NCDs.(3) The definition of HL mentions that there are multiple dimensions of the concept: functional, interactive and critical. Functional HL refers to the basic skills of reading and writing necessary for everyday situations. Interactive HL refers to advanced cognitive, literacy, and social skills needed to extract information from different forms of communication and apply it to different contexts. Critical HL refers to more advanced cognitive and social capabilities needed for critically appraising information and using it for greater control over life events and different situations that include the community.(4)

Nutrition is a major influence on the environment, physical and mental development since the first years of life.(5) In Romania, nutrition transition has taken place due to urbanization and rapid socio-economic changes and has resulted in a more westernized dietary pattern, especially among children and adolescents. This general shift in children's diet is characterized by low consumption of fruit and vegetables, fibre rich foods and dairy products, as well as high consumption of fatty, sugary and convenience foods.(6)

Food and nutrition literacy (FNLIT), both specific forms of HL, have become increasingly important concepts in promoting health. $(7,8)$ Improving not only the eating habits of the population, but also the knowledge and skills related to food, requires an understanding of the social context, but also individual and nutrition-related skills.(9)

Eating habits during childhood may influence preferences and practices in later life, and some evidence suggests fair to moderate follow-up of childhood to adolescence habits. Good nutrition helps improve children's well-being and learning potential, thereby contributing to better school performance.(10) WHO has identified schools as the ideal place to educate children and adolescents efficiently in developing healthy eating habits and accepting a healthy lifestyle throughout their lives.(11) Nutritional education in schools should address the needs and interests of school children, teachers and schools, be relevant to the purpose of the programmes, take into account the current learning of individuals, but also be appropriate from a cultural perspective. To succeed, nutrition education must be incorporated into the curriculum that actively involves teachers, family and other professionals in the community. $(5,10)$

FNLIT promotion is derived from its relevance in the context of school. However, the lack of documented policies and interventions in the field of FNLIT is a problem, in countries like Romania.

The promotion of FNLIT in children seems to be one of the most important elements in the improvement of healthy dietary pattern and prevention of diet-related NCDs for several reasons. $(7,8,12,13)$

${ }^{1}$ Corresponding author: Anca-Georgiana Gologan, Str. Iuliu Moldovan, Nr. 18, Cluj-Napoca, România, E-mail: georgiana.ispas@publichealth.ro, Phone: +40744992155

Article received on 03.08.2021 and accepted for publication on 27.08.2021 


\section{PUBLIC HEALTH AND MANAGEMENT}

\section{AIM}

The present research consisted of a pilot study of a nutrition intervention. The pilot study was in the form of a video and in-class activities with teachers trained to present the developed curriculum and materials. This intervention provides a choice of teaching media in nutrition education at schools instead of using the usual textbook.

This study aims to determine the effectiveness of a nutrition education programme implemented among lower secondary school children from two schools in Romania by investigating whether students tended to score higher in several FNLIT domains at post-test compared to the pre-test average. The present research results could help improve health and nutrition literacy and encourage school children to practice good eating habits in their daily lives.

\section{MATERIALS AND METHODS}

\section{Study design}

The present research uses a quasi-experimental approach with pre/post assessment involving 127 middle school students from two large municipalities from Romania. This pilot study was organized in 2 phases. During the first phase, the intervention programme's teaching materials (video and in-class activities) were developed and tested on a convenience sample of school children and teachers were recruited to pilot test the intervention in their class. In the second phase, an intervention was implemented for an average of three consecutive weeks at the two intervention schools. The study protocol was approved by the Public Health Department Institutional Review Board at Babeș-Bolyai University, Cluj-Napoca, decision number 2020201016- 004

\section{RESULTS}

Socio-demographic characteristics

Background characteristics of the school children that participated in the assessments and intervention are summarized in table no. 1 . The mean age of schoolchildren was 11.94 years $(\mathrm{SD}=0.77)$. The mean days between pre- and post-intervention assessment was 22.77 days ( $\mathrm{SD}=13.77)$. More than half of the students were in 5 th grade $(54.3 \%)$, the rest were from the sixth grade.

\section{Food and nutrition literacy at baseline}

Food and nutrition literacy (FNLIT) among the school children were analysed pre-intervention at the cognitive and skills domains. Regarding the cognitive domain, one of two students has a high understanding of information about nutrition on a food label $(30.7 \%$ usually and $23.6 \%$ always understanding). A high percentage of children $(59.1 \%)$ have good knowledge about what unhealthy eating means. Approximately $68 \%$ agree that high processed meat may cause obesity and a majority of $87.4 \%$ consider the expiration dates for food products are important. Regarding the functional domain questions, $45.7 \%$ of the children declared they never eat a variety of fruits and vegetables daily, while only a percentage of $1.6 \%$ of the sample reported always eating various fresh produce. More than half of school children can rely on getting nutrition-related information from their caregivers $(64.6 \%$ of the sample reported that they usually or always could receive advice). A low proportion of children (5.5\%) bring healthy snacks to school, while more than half of the sample $(52 \%)$ do not practice this healthy habit on a regular daily basis. More than half of the sample (65\% of students) are not actively involved in handling and preparing fruits and vegetables. A high percentage $(35.4 \%)$ of children admit that they only sometimes have the will to resist unhealthy foods and know how to choose healthy ones, and only $20.5 \%$ reported mastering this ability. If the family offers unhealthy snacks, more than half of the students can refuse them, and only $20 \%$ of students accept unhealthy snacks to bring to school. More than $55 \%$ of students categorically refuse unhealthy food proposals from family or friends. A large percentage $(61.4 \%)$ declares that they will intervene to recommend a change in life expectancy if a family member were obese or had a high-fat diet. When shopping is done under parental supervision, $57.5 \%$ of students say they always choose healthy foods that are not expired and have appropriately documented nutritional labels. More than $30 \%$ of students reported they never or seldom try new vegetables from the sample, and only $16.5 \%$ declare that they always try new vegetables. Conversely, a high portion of students reported a high level of label literacy $(79.5 \%)$.

\section{Changes in food and nutrition literacy}

Wilcoxon signed-ranks tests were conducted to compare FNLIT among students analysed before and after the intervention.

At the understanding subscale for the question "I easily understand the information about nutrition (e.g. calories, sugar, protein, etc.) on a food label", there was a decrease of $4 \%$ among those who answered never \& seldom, also a decrease was noticed for those who reported sometimes $(15 \%)$, and for usually \& always understanding of nutritional information, the percentage increased significantly by $19 \%$, compared to pre- and post-intervention results. The question "I understand information and recommendations about healthy eating for children my age (for example, those I receive from nutritionists, the internet, brochures etc.)" showed an increase in the percentage of those who never \& rarely understand $(6.3 \%)$, postintervention, a decrease of $15 \%$ for those who responded sometimes and an increase of only $8.7 \%$ for those who usually \& always understand nutritional information and recommendations, post-intervention.

At the interactive subscale, for the question "I have enough willpower to resist unhealthy foods (e.g., fast food, pizza, carbonated drinks, etc.) and I know how to choose healthy foods", a slight decrease of $1.6 \%$ was observed for those who said that never \& seldom have the strength to resist, those who answered sometimes, recorded a significant decrease, after intervention of $15.7 \%$, and those who said that they usually \& always have enough will increased by 17.3 percent compared to the pre-intervention survey.

When asked - I can easily say "No" to any unhealthy eating suggestions from my friends -, 18 post-intervention participants chose never \& seldom compared to 29 postintervention students $(8.6 \%)$ for those who answer sometimes, the percentages remained similar, pre and post intervention, and there was a slight increase after intervention for those who answered that usually \& always say 'no' to unhealthy foods with a percentage of $9.4 \%$.

At the critical subscale, the question was about the desire to try new vegetables; there was a post-intervention decrease of $7.1 \%$ for those who answered never \& seldom, a slight decrease for those who answered sometimes (5.5\%) and an increase of over $12 \%$ for those who usually \& always want to try new vegetables.

At the label literacy subscale, which verified the reading and understanding capabilities of the information on the nutritional labels, at the post-intervention, 26 participants declared that they do not have a good understanding of this information $(21 \%)$, and at post-intervention their number was reduced to 16 students, obviously those who answered affirmatively to the understanding of the labels, they registered an increase of just over 7 percent in the post-intervention interview. Standard deviation (SD), mean (M) and confidence interval (CI) were used for the statistical analysis of the descriptive variables. 
Table no. 1 - Food and nutrition literacy results in the Romanian sample at the baseline

\begin{abstract}
Cognitive domain
Understanding: I

easily understand the

information about

nutrition (e.g. calories, sugar, protein, etc) on a food label.

Understanding: I

understand information

and recommendations

about healthy nutrition

for children my age

(e.g. those I receive

from nutritionists, the

internet, brochures, etc.).
\end{abstract}

Knowledge: Eating salty snacks, sugar, sweets or chocolate is harmful to your health. Knowledge:

Consumption of salami and sausage that are high in fat may cause

$$
\text { obesity }
$$

Knowledge: When shopping, how important are production and expiration dates for you?

Skill domain

Functional: I eat a variety of vegetables (e.g., lettuce, cabbage, tomatoes, carrots, etc.) and fruits every day Functional: If I have any questions about

food and nutrition issues, I'm able to get information and advice from parents, teachers, etc.

Functional: I bring healthy snacks to school.

Functional: I wash and prepare fruits and

vegetables by myself. Interactive: I have enough will power to resist unhealthy foods (e.g., fast food, pizza, carbonated drinks, etc.) and I know how to

choose healthy foods. Interactive: If my parents or family prepare unhealthy snacks (e.g., chips, fruit roll-ups, corn snacks, etc.) for me to take to school, I accept them Interactive: I can easily say "No" to any unhealthy eating suggestions from my friends.

Interactive: If my family were overweight and eating a high-fat diet, I would tell them to change their eating habits. Preintervention Postintervention Preintervention Postintervention

\begin{tabular}{cc}
\multicolumn{2}{c}{ Never \& Seldom } \\
$14,20 \%$ & $10,20 \%$ \\
18 & 13 \\
$4,70 \%$ & $11 \%$ \\
6 & 14 \\
&
\end{tabular}

Strongly agree \& Agree

$78,80 \%$

$85 \%$

$\begin{array}{cc}99 & 108 \\ 68,50 \% & 73,20 \%\end{array}$

87

Not important
$92,90 \%$

118

$92,10 \%$

117

\begin{tabular}{cc}
\multicolumn{2}{c}{ Never \& Seldom } \\
$77,20 \%$ & $74,80 \%$ \\
98 & 95 \\
$10,20 \%$ & $9,40 \%$ \\
&
\end{tabular}

13

12

$\begin{array}{cc}15,70 \% & 16,50 \% \\ 20 & 21 \\ 18,10 \% & 16,50 \% \\ 23 & 21 \\ 18,10 \% & 16,50 \%\end{array}$

23

21

$56,70 \%$

$63,80 \%$

81

72

$22,80 \%$

$14,20 \%$

29

18

$10,20 \%$

$6,30 \%$

8

$$
31,50 \% \text { Sometimes }
$$

40

$25,20 \%$

32

Neither agree nor disagree

$17,30 \% \quad 9,40 \%$

22

$18,90 \%$

12

24

$15 \%$

19

$1,60 \% \quad$ Neutral $2,40 \%$

$$
2
$$

3

$15,70 \%$ Sometimes $16,50 \%$

$25,20 \%$

32

$32,30 \%$
41
$16,50 \%$
21

$35,40 \%$

45

$21,30 \%$

$15,70 \%$

20

$18,90 \%$

24

$15,70 \%$

20

$19,70 \%$

25

27

$18,90 \%$

$18,10 \%$

23

$10,20 \%$

13

$10,20 \%$

13
Preintervention Postintervention

Usually \& Always

$54,30 \%$

$73, \%$

69

93

$70,10 \%$

$78,80 \%$

89

100

Disagree \& strongly distragree $4,70 \%$

$5,50 \%$

$\begin{array}{cc}6 & 7 \\ 12,60 \% & 11,80 \%\end{array}$

16

15

Somewhat \& very important $5,50 \% \quad 5,50 \%$

7

7

Usually \& Always $7,10 \%$

$8,70 \%$

9

11

$64,20 \%$

$73,20 \%$

64,6

93

\section{$52 \%$}

$65,40 \%$

83

$46,50 \%$

59

$21,30 \%$

$19,70 \%$

27

$58,30 \%$

$67,70 \%$

74

86

$79,50 \%$

101

$83,50 \%$

106 


\section{PUBLIC HEALTH AND MANAGEMENT}

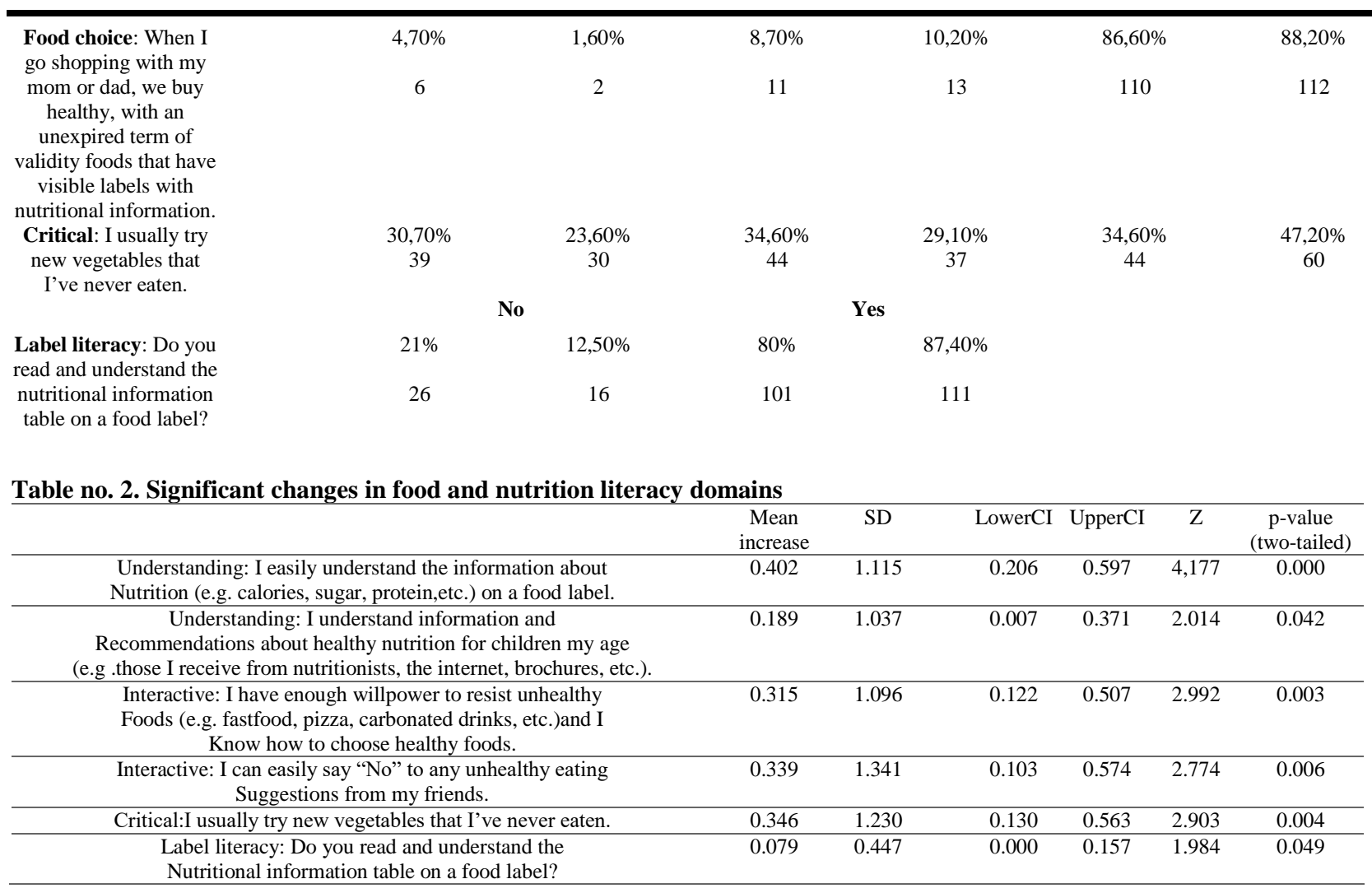

Standard deviation shows how much variation or dispersion exists from the average (mean), or expected value. The most common expression for the mean of a statistical distribution with a discrete random variable is the mathematical average of all the terms. The confidence interval is the range of values that you expect your estimate to fall between a certain percentage of the time if you run your experiment again or re-sample the population in the same way. In essence, a confidence interval is a range that we expect, with some level of confidence, to include the actual value of population mean. $(20,21)$

The results are highlighted in table no. 2, where the statistically significant changes following the intervention in understanding, interactive, critical or label literacy are presented (CI 95\%). There was a significant increase in the mean scores for the understanding of the information about nutrition on food labels $(\mathrm{p}=0,000)$ before intervention $(\mathrm{M}=3.61, \mathrm{SD}=1.047)$ and after the intervention $(\mathrm{M}=4.02, \mathrm{SD}=1.098)$.

There was also a significant difference in the score for the understanding of recommendations about healthy nutrition $(p=0.042)$ with a mean score of 4.01 in the sample preintervention $(\mathrm{SD}=0.921)$, respectively a mean score of 4.2 postintervention $(\mathrm{SD}=1.084)$. In the interactive scores, there was a significant increase of mean scores $(\mathrm{p}=0.003)$ before the intervention $(\mathrm{M}=3.4, \mathrm{SD}=1.170)$ and after intervention $(\mathrm{M}=3.72$, $\mathrm{SD}=3.72$ ). The students, post-intervention, reported significant greater willpower to resist unhealthy foods or to choose healthy foods. There was a significant difference $(p=0,006)$ in the interactive scores related to student's attitude in saying no to unhealthy foods.

\section{DISCUSSIONS}

An unhealthy diet accompanied by overweight continues to be one of the most important global problems from all perspectives (health, social and economic). Changes in eating behaviour and the prevention of childhood obesity through specific interventions are urgently needed to reduce the incidence of chronic no communicable diseases.(4) A low level of nutritional and food literacy is a barrier to a nutritionally balanced diet among school-age children. Previous research has shown that high literacy is associated with an increased consumption of fruits and vegetables.

The aim of the present study was to determine the effectiveness of a nutrition education programme implemented among secondary school children from two schools in Romania. The nutrition education programme conducted in this study increased students' food and nutrition literacy on the understanding, interactive, critical, and label literacy subscales of FNLIT. Our results showed that post-assessment students reported better understanding of information about nutrition and information and recommendations about healthy nutrition for children their age provided from nutritionists, or the internet as a result of the interventions. Research in the field shows similar results, in which children reported an enhanced nutrition literacy on multiple literacy subscales following school based nutrition education programmes.(14-17)

Our results show that an approach combining educational video materials coupled with in-class exercises can lead to not only increased knowledge but also increased dietary habits such as trying new vegetables and resisting different types of unhealthy foods, even after a relatively short intervention for a mean period of three weeks. These results are supported by the existent literature reviews in the field, which demonstrated that school-based interventions are able to affect attitudes, knowledge, behaviour, especially if they consider an environmental approach and actively involve the students.(17)

Moreover, using multiple learning materials, such as educational videos and in-class exercises, proved to be useful in increasing students' food and nutrition literacy. Research in the nutrition field shows that multi-component interventions have the potential to create lasting improvements especially in the school environment (17) and video materials found to be effective in increasing knowledge about healthy food and nutrition in children. $(18,19)$ Even though the study period was 


\section{PUBLIC HEALTH AND MANAGEMENT}

only 3 weeks, significant progress was noted among the participants.

Using games and promoting health is a new research area. Existing publications confirm that games tool can be beneficial to health. Scientific research and case studies have shown that games and stadiums have an impact on health and should be considered as essential elements in the health domain.(22) Gamification is a design strategy that attempts to reproduce the interactive powers of games and imitate key gameplay features in non-game contexts without designing a complete game.(23) Therefore, developers should pay attention to health apps that not only track user health, but also motivate the user to correct unhealthy behaviours and help improve their daily lives.

However, our study did not find significant results on the functional, knowledge and food choice FNLIT sub-scales These results might be partly explained by the young age of the children, since they are not yet independent on buying their own food or being responsible for the food choices for school or in general. Also, our intervention focused mostly on improving understanding and basic knowledge of nutrition concepts and did not have a specific focus on developing skills related to food choice. The length of the interventions can be another factor explaining our results, since our intervention was relatively short, for a mean period of three weeks, while most of the interventions that have significant results have duration of several months, with longer interventions having better results.(17)

Secondly, this study can be a starting point for policy makers and those responsible for developing the educational curriculum in order to implement practical educational programs, as well as teaching strategies to improve the level of nutritional literacy and food among schoolchildren.(7) But one of the essential problems in implementation is the lack of a budget allocated to nutrition education, as well as staff dedicated to this purpose.

Moreover, research in the field also showed that an increase in the understanding of nutritional concepts does not necessarily bring positive changes in making healthy food choices, since it takes longer time for people to change their food eating patterns and choices.(14)

\section{CONCLUSIONS}

Results of the present study show that a multicomponent intervention using both video materials and in class exercises and involving teachers as trainers had significant results in terms of improving food and nutrition literacy in secondary school children from Romania. Even if the intervention period had a relatively short period of 3 weeks improvements in understanding, interactive food and nutrition literacy, critical food and nutrition literacy, and label literacy were observed. These results have the potential of informing future interventions to improve food and nutrition literacy for school aged children. Future research should focus on expanding the intervention should be expanded in more schools and have a longer duration. The present results also stand as a basis for future policies aiming to include food and nutrition education in the school curricula as a tool for improving healthy eating and preventing NCDs associated with nutrition.

\section{REFERENCES}

1. World Health Organization (WHO). Noncommunicable Disease Country Profiles 2018. Geneva: World Health Organization (WHO); 2018. p. 1-157.

2. Rus VA. The Role of Healthy Diet and Lifestyle in Preventing Chronic Diseases. J Interdiscip Med. 2019;4(2):57-8
3. Heine M, Lategan F, Erasmus M, Lombaard CM, Mc Carthy N, Olivier J, et al. Health education interventions to promote health literacy in adults with selected noncommunicable diseases living in low-to- middle income countries: A systematic review and meta-analysis [Internet]. Journal of Evaluation in Clinical Practice; 2021 Mar 22.

4. Nutbeam D. The evolving concept of health literacy. Soc Sci Med. 2008;67(12):2072-8

5. Krause C, Sommerhalder K, Beer-Borst S, Abel T. Just a subtle difference? Findings from a systematic review on definitions of nutrition literacy and food literacy. Health Promot Int. 2016;33(3):378-89.

6. Constanța H, Brîndușe L. Evaluarea Stării De Nutriţie A Copiilor Din Ciclul Primar Conform Metodologiei Oms Prin Participarea La Proiectul "European Childhood Obesity Surveillance Initiative (Cosi)" Raport. Bucharest; 2017.

7. Doustmohammadian A, Omidvar N, Shakibazadeh E. School-based interventions for promoting food and nutrition literacy (FNLIT) in elementary school children: A systematic review protocol. Syst Rev. 2020;9(1):1-8.

8. Doustmohammadian A, Omidvar N, KeshavarzMohammadi N, Eini-Zinab H, Amini M, Abdollahi M, et al. Low food and nutrition literacy (FNLIT): A barrier to dietary diversity and nutrient adequacy in school age children. BMC Res Notes 2020;13(1):1-8.

9. Vidgen HA, Gallegos D. Defining food literacy and its components. Appetite. 2014 May 1;76:50-9.

10. Pérez-Rodrigo C, Aranceta J. School-based nutrition education: lessons learned and new perspectives. Public Health Nutr. 2001;4(1a):131-9.

11. Programme for Nutrition and Food Security WHO Regional Office for Europe Copenhagen. Food andnutrition policy for schools. World Heal Organ. 2006;58.

12. Doustmohammadian A, Omidvar N, Shakibazadeh E. School-based interventions for promoting food and nutrition literacy (FNLIT) in elementary school children: A systematic review protocol. Syst Rev. 2020;9(1):1-7.

13. Doustmohammadian A, Omidvar N, KeshavarzMohammadi N, Abdollahi M, Amini M, Eini-Zinab H. Developing and validating a scale to measure Food and Nutrition Literacy (FNLIT) in elementary school children in Iran. PLoS One. 2017;12(6):1-18.

14. Ruzita AT, Wan Azdie MAB, Ismail MN. The effectiveness of nutrition education programme for primary school children. Malays J Nutr. 2007;13(1):45-54.

15. Sharif Ishak SIZ, Chin YS, Mohd Taib MN, Chan YM, Mohd Shariff Z. Effectiveness of a school-based intervention on knowledge, attitude and practice on healthy lifestyle and body composition in Malaysian adolescents. BMC Pediatr. 2020;20(1):1-13.

16. Jung T, Huang J, Eagan L, Oldenburg D. Influence of school-based nutrition education program on healthy eating literacy and healthy food choice among primary school children. Int J Heal Promot Educ. 2019 Mar 4;57(2):67-81.

17. Chaudhary A, Sudzina F, Mikkelsen BE. Promoting healthy eating among young people - a review of the evidence of the impact of school-based interventions. Nutrients. 2020;12(9):1-34

18. Hieftje K, Edelman EJ, Camenga DR, Fiellin LE. Electronic media-based health interventions promoting behavior change in youth: A systematic review. JAMA Pediatr. 2013;167(6):574-80.

19. Folkvord F, Anschütz D, Geurts M. Watching TV Cooking Programs: Effects on Actual Food Intake Among Children. J Nutr Educ Behav. 2020;52(1):3-9. 


\section{PUBLIC HEALTH AND MANAGEMENT}

20. Barde MP, Barde PJ. What to use to express the variability of data: Standard deviation or standard error of mean? Perspect Clin Res; 2012.

21. Pyrczak F, Oh D. Making Sense of Statistics - A Conceptual Overview. 7th ed. Routledge; 2018. p. 120-134.

22. Klasnja P, Consolvo S, Pratt W. How to evaluate technologies for health behavior change in HCI research. Conf Hum Factors Comput Syst - Proc. 2011;3063-72.

23. Deterding S, O'Hara K, Sicart M, Dixon D, Nacke L. Gamification: Using game design elements in non-gaming contexts. Conf Hum Factors Comput Syst - Proc. 2011;2425-8. 